\title{
How far can we trust published TESS periods?
}

\author{
Z. Mikulášek ${ }^{1}$ and M. Skarka ${ }^{1,2}$ \\ 1 Department of Theoretical Physics an Astrophysics, Masaryk University, \\ Kotlársská 2, 61138 Brno, The Czech Republic (E-mail: \\ mikulas@physics.muni.cz) \\ 2 Astronomical Institute of the Czech Academy of Sciences \\ 25165 Ondřejov, The Czech Republic
}

Received: November 6, 2019; Accepted: February 4, 2020

\begin{abstract}
Possible inaccuracies in the determination of periods from shortterm time series caused by disregarding light curve instrumental effects are documented. As an example, we present a Lomb-Scargle period analysis of a simulated TESS-like light curve.
\end{abstract}

Key words: stars: variable - period analysis - TESS data

\section{Introduction}

TESS data are now one of the most popular sources of information about variable stars, including their periods. However, TESS data suffer from two shortcomings that significantly corrupt the results of period analyses with standard tools. The data are usually strongly affected by instrumental trends of various kinds, and secondly, they are obtained in non-standard short time intervals (for example 27 days for TESS) that are often comparable to the periods themselves. The majority of such days-long periods and their uncertainties are mere artifacts of the method used to determine them. At best, they are just estimates.

The most commonly used method is the so-called Lomb-Scargle method (Press \& Rybicki, 1989) or its sophisticated versions, enabling to take into account the weights of individual measurements and to estimate the amplitudes of the found frequency peaks (Mikulášek et al., 2015). All of these methods give identical results because they are based on fitting the observation series with a first-order harmonic polynomial model using the least squares method.

The following demonstration based on a simulated periodic light curve resembling that of a rotating chemically peculiar stars with a period of 2.7 days (see Fig. 1) shows possible pitfalls of standard processing of these types of data. 


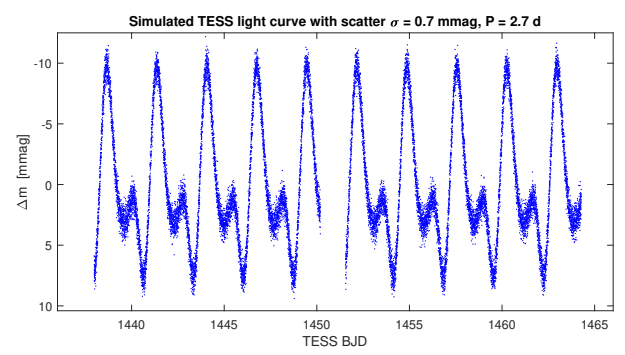

Figure 1. Simulated light curve without trends represented by 17925 points.

\section{Simulation of "TESS-like" data of a hypothetical CP star}

Chemically peculiar (CP) stars are rotating stars with extensive photometric spots on their surface. The observed light changes of such objects are strictly periodic and can be described by a low-degree harmonic polynomial. For our example, we chose a two-wave curve represented by a third-degree harmonic polynomial, described by five parameters (Fig. 2) of the phase function, $\vartheta$ (for details see Mikulášek et al., 2008), $F(\vartheta, \mathbf{a}), P=2.7 \mathrm{~d}$, with a maximum at phase $\varphi=0$. We have

$$
\vartheta=\frac{t-M_{0}}{P}=E+\varphi, \quad M_{0}=1450+P \Delta \varphi, \quad E=\operatorname{IP}(\vartheta), \quad \varphi=\operatorname{FP}(\vartheta),
$$

with

$$
\begin{gathered}
F(\vartheta, \mathbf{a})=a_{1} \cos (2 \pi \vartheta)+a_{2} \cos (4 \pi \vartheta)+a_{3} \cos (6 \pi \vartheta)+ \\
+a_{4}[2 \sin (2 \pi \vartheta)-\sin (4 \pi \vartheta)]+a_{5}[3 \sin (2 \pi \vartheta)+6 \sin (4 \pi \vartheta)-5 \sin (6 \pi \vartheta)]
\end{gathered}
$$

where $t$ is the TESS BJD time of the observation ( $t=\mathrm{BJD}-2457000), M_{0}$ is the TESS BJD time of the initial light curve maximum, $\Delta \varphi$ is an optional initial phase parameter allowing for a horizontal shift of the simulated light curve, $E$ is the integer epoch and $\varphi$ the phase. IP and FP indicate the integer and fractional part of a variable. We also have $a_{1}=-5, a_{2}=-4.5, a_{3}=-0.5, a_{4}=-0.67$, and $a_{5}=-0.17 \mathrm{mmag}$. The simulated TESS light curve is represented by 17925 points obtained at a cadence of 2 minutes (see Fig. 1). Upon demand the curve can include trends and Gaussian scatter.

\section{The role of the initial phase $\Delta \varphi$}

The amplitude frequency spectrum of the simulated light curve shows, as expected, three dominant, equidistant peaks with central frequencies $f_{1}, f_{2}$, and $f_{3}$ (Fig. 2), each carrying the period information: $1 / f_{1}=2 / f_{2}=3 / f_{3}=P$. 


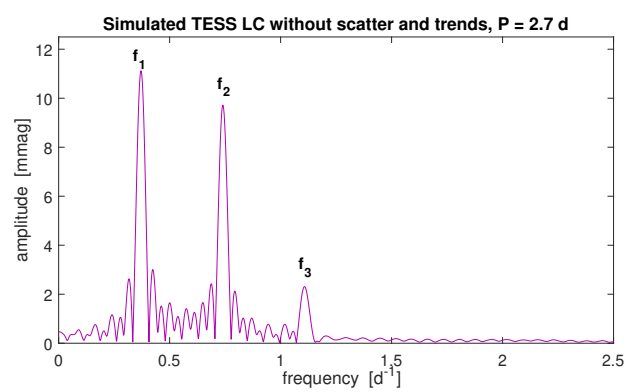

Figure 2. The amplitude periodogram of the simulated light curve without scatter and trends for $\Delta \varphi=0$.

However, this is fulfilled only approximately. If we limit ourselves to two peaks, then for $\Delta \varphi=0$ we get $P_{1}=1 / f_{1}=2.7070(10) \mathrm{d}$, and $P_{2}=2 / f_{2}=2.6977(8)$ d. The deviation from the baseline period $P=2.7 \mathrm{~d}$ thus is evident and far exceeds the limits given by the uncertainty of the positioning of the frequency peaks. Why such difference? Other simple period finders give the same results.

The discrepancy would only disappear if the light curves were purely sinusoidal without higher harmonics. If the light curves deviate from this ideal, the so-called 'periods' determined are not real periods, but only parameters found by regression with an inadequate model that differs (sometimes flagrantly) from the real frequency pattern. Fig. 3 shows that the values of those 'periods' found in the periodograms are a complex periodic function of the initial phase $\Delta \varphi$, whose amplitude is unacceptable in comparison with the formal determination of the uncertainty.

\section{Influence of light curve trends}

Both TESS and Kepler observations are strongly affected by aperiodic instrumental trends (Hümmerich et al., 2018; Mikulášek et al., 2019). Neglecting these has a devastating effect on period analysis (Fig.4). Appropriate detrending of the observed light curves is highly desirable if we want to fully realize the benefits of the unprecedented accuracy of satellite photometry.

\section{Modelling of light curves with trends}

When applying the Lomb-Scargle method, we have to subtract from each data point the mean value of the data set. Subsequently, the data are fitted by the simplest possible model for a periodic light curve, i.e. a linear combination of a pair of harmonic functions $F=a_{1} \cos (2 \pi f)+a_{2} \sin (2 \pi f)$, where the amplitude $A=\sqrt{a_{1}^{2}+a_{2}^{2}}$ is a function of the frequency, $f$, and plotted on the $A(f)-f$ 


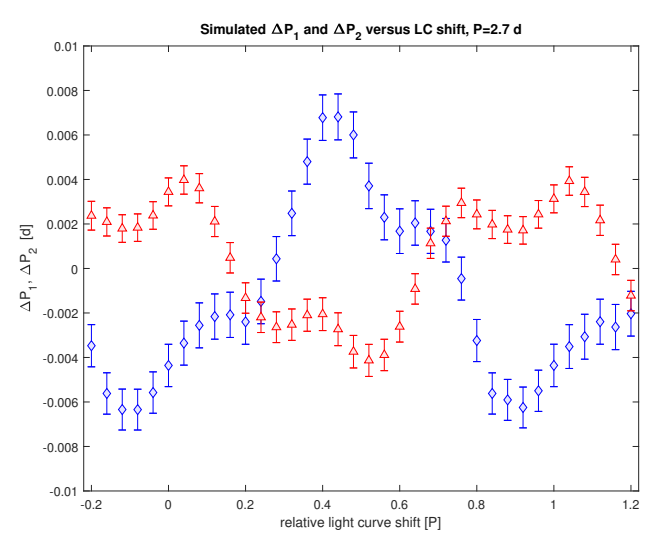

Figure 3. $\Delta P_{1}=P_{1}-P$ (blue diamonds) and $\Delta P_{2}=P_{2}-P$ (red triangles) differences as a function of the light curve shift $\Delta \varphi$.

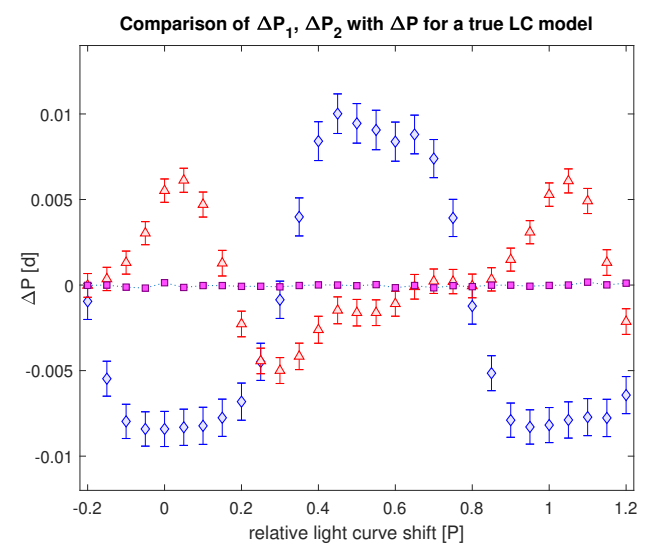

Figure 4. The comparison of $\Delta P_{1}$ (blue diamonds) and $\Delta P_{2}$ (red triangles) differences and true model ones (pink squares) for a non-detrended light curve as a function of the LC shift $\Delta \varphi$.

periodogram. The extrema of the plotted function are then searched for and interpreted. Uncertainties of the frequencies determined by least-squares tools are generally defective (most often they are underestimated) simply because the conditions for applying the least squares method are not fulfilled. ${ }^{1}$

It is apparent from the foregoing examples that the use of conventional lowparametric models is not sufficient for the description of high-precision real light

${ }^{1}$ Residuals do not have a normal distribution, consecutive residuals are not independent, etc. 
curves provided by modern instruments, in particular satellites. It is, therefore, essential to use more advanced models to simultaneously fit a phase curve with a harmonic polynomial of at least third degree, while modeling trends by dividing the light curve into segments and describing each with a polynomial of the appropriate degree (Mikulášek et al., 2019; Hümmerich et al., 2018). Phase curves and trend models should be tailored to the actually observed light curves. The models should be functions of the parameters of the ephemeris of stellar periodicity, especially the reference time of the basic extremum, the mean period (or periods) and their time derivatives (Mikulášek, 2015, 2016).

Modern tools such as a chi-square approach, robust regression, bootstrapping, etc., can be used to find parameters (including period/periods) and their uncertainties. In Fig. 4 results of such a rigorous procedure are compared with the results obtained by standard trivial period analysis procedures. The comparison speaks for itself and no further comments are needed.

\section{Conclusions}

- Short-term observational series corrupted by instrumental trends are not the easiest observational material for accurate determination of periods.

- Lomb-Scargle and its derivatives are sources of serious errors both in the determination of periods and their uncertainties.

- In the period analysis of TESS data, the differences between the obtained and the real periods can be so huge that we are not able to predict the correct phase at a particular moment in time that differs more than a few months from the actual time of observation.

A rigorous solution to the problem is to move to realistic models of light curves, including a true description of phase curve/curves as well as instrumental trends. Only then will we fully use the information potential of short-term sets of observational data.

Acknowledgements. This work has been supported by grant GAČR 18-05665S and OP VVV Project Postdoc@MUNI (No. CZ.02.2.69/0.0/0.0/16_027/0008360).

\section{References}

Hümmerich, S., Mikulášek, Z., Paunzen, E., et al., The Kepler view of magnetic chemically peculiar stars. 2018, Astron. Astrophys., 619, A98, DOI: 10.1051/0004$6361 / 201832938$

Mikulášek, Z., Phenomenological modelling of eclipsing system light curves. 2015, Astron. Astrophys., 584, A8, DOI: 10.1051/0004-6361/201425244 
Mikulášek, Z., Monitoring of rotational period variations in magnetic chemically peculiar stars. 2016, Contributions of the Astronomical Observatory Skalnate Pleso, 46, 95

Mikulášek, Z., Krtička, J., Henry, G. W., et al., The extremely rapid rotational braking of the magnetic helium-strong star HD 37776. 2008, Astron. Astrophys., 485, 585, DOI: 10.1051/0004-6361:20077794

Mikulášek, Z., Paunzen, E., Netopil, M., \& Zejda, M., New Tools for Finding and Testing Weak Periodic Variability. 2015, in Astronomical Society of the Pacific Conference Series, Vol. 494, Physics and Evolution of Magnetic and Related Stars, ed. Y. Y. Balega, I. I. Romanyuk, \& D. O. Kudryavtsev, 320

Mikulášek, Z., Zejda, M., Hümmerich, S., et al., Monitoring Period Variations of Variable Stars using Precise Photometric Surveys. 2019, in IAU Symposium, Vol. 339, Southern Horizons in Time-Domain Astronomy, ed. R. E. Griffin, 110-113

Press, W. H. \& Rybicki, G. B., Fast Algorithm for Spectral Analysis of Unevenly Sampled Data. 1989, Astrophys. J., 338, 277, DOI: 10.1086/167197 